\title{
Tracking Stationary Extended Objects for Road Mapping using Radar Measurements
}

\author{
Christian Lundquist, Umut Orguner and Thomas B. Schön \\ Division of Automatic Control \\ Linköping University \\ SE-581 83 Linköping, Sweden \\ Email: \{lundquist, umut, schon\}@isy.liu.se
}

\begin{abstract}
It is getting more common that premium cars are equipped with a forward looking radar and a forward looking camera. The data is often used to estimate the road geometry, tracking leading vehicles, etc. However, there is valuable information present in the radar concerning stationary objects, that is typically not used. The present work shows how stationary objects, such as guard rails, can be modeled and tracked as extended objects using radar measurements. The problem is cast within a standard sensor fusion framework utilizing the Kalman filter. The approach has been evaluated on real data from highways and rural roads in Sweden.
\end{abstract}

\section{INTRODUCTION}

For a collision avoidance system it is imperative to have a reliable map of the environment surrounding the ego vehicle. This map, consisting of both stationary and moving objects, has to be built in real time using measurements from the sensors present in the ego vehicle. This is currently a very active research topic within the automotive industry and many other areas as well. Great progress has been made, but much remains to be done. Current state-of-the-art when it comes to the problem of building maps for autonomous vehicles can be found in the recent special issues [1]-[3] on the 2007 DARPA Urban Challenge. In these contributions measurements from expensive and highly accurate sensors are used, while we in the present paper utilize measurements from off-the-shelf automotive radars.

Obviously, these stationary radar measurements are not enough to fully explain the road borders. However, as we will see, there is surprisingly much information present in these measurements.

In this contribution we consider the problem of estimating the position and shape of stationary objects in front of the vehicle, making use of echoes from a standard automotive radar. Hence, there is no need to introduce any new sensors, it is just a matter of making better use of the sensor information that is already present in a modern premium car. We represent the stationary objects as

- points, with sources such as delineators or lampposts or

- lines, where measurements stem from e.g. guard rails or concrete walls.

The lines are modeled as extended objects, since an object is denoted extended whenever the object extent is larger than the sensor resolution. Put in other words, if a object should be classified as extended does not only depend on its physical size, but also on the physical size relative to the sensor resolution. Extended object tracking is extensively described in e.g. [4], [5] and it has received quite recent attention in [6], [7] where Monte Carlo methods are applied and in [8] which is based on random matrices.

The problem of road border estimation has been investigated in the literature mostly in the last decade. The approaches presented mainly differ in their models for the road borders and the different types of sensors used in the estimation. The third order approximation of the two sided (left and right) "clothoid model" has been used in connection with Kalman filters in [9] and [10] for laser scanner measurements and radar measurements respectively. In [11], Lundquist and Schön proposed two road border models, one of which is very similar to [10] and used a constrained quadratic program to solve for the parameters. A linear model represented by its midpoint and orientation (one for each side of the road) is utilized in [12] with ladar sensing for tracking road-curbs. Later, [13] enhanced the results of [12] with the addition of image sensors. A similar extended Kalman filtering based solution is given in [14], where a circular road border modeling framework is used. Recently, the particle filters (also referred to as condensation in image and video processing) have been applied to the road border estimation problem in [15] with an hyperbolic road model.

The present solution extends an already existing sensor fusion framework [16], which among other things provides a good road geometry estimate. This framework improves the raw vision estimate of the road geometry by fusing it with radar measurements of the leading vehicles and information from various proprioceptive sensors. The idea is that the motion of the leading vehicles reveals information about the road geometry [17]-[19]. Hence, if the leading vehicles can be accurately tracked, their motion can be used to improve the road geometry estimates. Furthermore, we used a solid dynamic model of the ego vehicle allowing us to further refine the estimates by incorporating several additional sensor measurements from the CAN bus. The resulting, rather simple, yet useful map of the environment surrounding the ego vehicle consists in

- Road geometry, typically parameterized using road curvature and curvature rate.

- Position and velocity of the leading vehicles.

- Ego vehicle position, orientation and velocity.

- Position and shape of stationary objects.

The stationary objects are tracked by casting the problem within a standard sensor fusion framework. Since we use a 
linear model and assume Gaussian noise we use the standard Kalman filter [20].

The approach has been evaluated on real data from highways and rural roads in Sweden. The test vehicle is a Volvo S80 equipped with a forward looking $77 \mathrm{GHz}$ mechanically scanned FMCW radar and a forward looking vision sensor (camera).

\section{Geometry AND Notation}

In [16] we provide a sensor fusion framework for sequentially estimating the parameters $l, \delta_{r}, c_{0}$ in the following model of the road's white lane markings,

$$
y^{E}=l+\delta_{r} x^{E}+\frac{c_{0}}{2}\left(x^{E}\right)^{2},
$$

where $x^{E}$ and $y^{E}$ are expressed in the ego vehicle's coordinate frame $E$. The angle between the longitudinal axis of the vehicle and the road lane is $\delta_{r}$, see Figure 1 . It is assumed that this angle is small and hence the approximation $\sin \delta_{r} \approx \delta_{r}$ is used. The curvature parameter is denoted by $c_{0}$ and the offset between the ego vehicle and the white lane is denoted by $l$.

In this paper we will use the planar coordinate transformation matrix

$$
R^{W E}=\left(\begin{array}{cc}
\cos \psi_{E W} & -\sin \psi_{E W} \\
\sin \psi_{E W} & \cos \psi_{E W}
\end{array}\right)
$$

to transform a vector, represented in the ego vehicle's coordinate frame $E$, into a vector, represented in the world reference coordinate frame $W$, where $\psi_{E W}$ is the angle of rotation from $W$ to $E$. We will refer to this angle as the yaw angle of the vehicle, and in order to simplify the notation we will use $\psi \triangleq \psi_{E W}$. The point $O_{W}$ is the origin of $W$ and $O_{E}$ is the origin of $E$ situated in the vehicles center of gravity. The geometric displacement vector $r_{O_{E} O_{W}}^{W}$ is the straight line from $O_{W}$ to $O_{E}$ represented with respect to frame $W$. The angles and distances are shown in Figure 1. Hence, a point $P^{E}$ represented in the ego vehicle coordinate frame $E$ is transformed to be represented in the world coordinate frame $W$ using

$$
P^{W}=R^{W E} P^{E}+r_{O_{E} O_{W}}^{W} .
$$

An observation $m$ will be referred to as a stationary object in the point $\mathcal{S}_{m}$. The radar in the ego vehicle measures the azimuth angle $\psi_{\mathcal{S} E}$ and the range $r=\left\|r_{\mathcal{S}_{m} O_{E}}^{E}\right\|_{2}$. These are transformed into Cartesian coordinates $\mathcal{S}_{m}^{E}=$ $\left[\begin{array}{ll}x_{\mathcal{S}_{m} O_{E}}^{E} & y_{\mathcal{S}_{m} O_{E}}^{E}\end{array}\right]^{T}$.

\section{EXTENDED OBJeCT MODEL}

In this section we introduce models for the tracked stationary object, i.e., points and lines. To take care of the lines a model with the object's shape, size, position and orientation is introduced.

\section{A. Motion Model of the Stationary Objects}

Stationary objects are modeled as points $\mathcal{P}$ or lines $\mathcal{L}$. A point $\mathcal{P}_{i}$ is represented using a position in the planar world coordinate frame $W$, according to

$$
\mathbf{x}_{\mathcal{P}_{i}} \triangleq\left[\begin{array}{ll}
x_{\mathcal{P}_{i} O_{W}}^{W} & y_{\mathcal{P}_{i} O_{W}}^{W}
\end{array}\right]^{T} .
$$

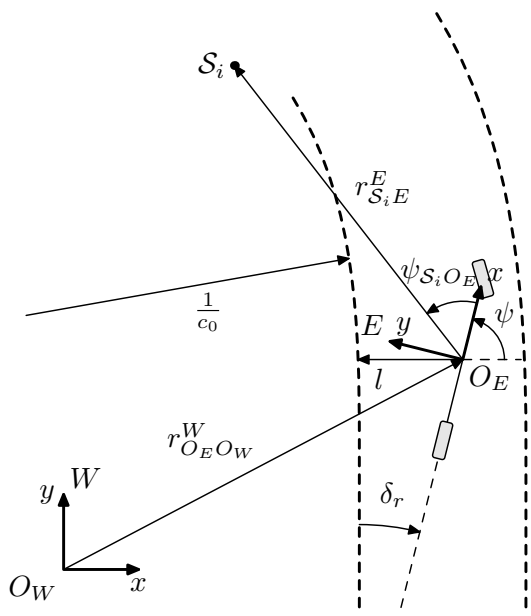

Figure 1. The ego vehicle's coordinate frame $E$ has its origin $O_{E}$ situated in the vehicle's center of gravity. A stationary object $\mathcal{S}_{i}$ is observed at a distance $\left\|r_{\mathcal{S}_{i} O_{E}}^{L}\right\|_{2}$ and an angle $\psi_{\mathcal{S}_{i} O_{E}}$ with respect to the radar. The angle between the vehicle's longitudinal axis and the road lane is $\delta_{r}$, the distance to the left lane marking is $l$ and the road curvature is $c_{0}$.

A line $\mathcal{L}_{j}$ is represented as a second order polynomial in its coordinate frame $L_{j}$

$$
y^{L_{j}}=a_{0}+a_{1} x^{L_{j}}+a_{2}\left(x^{L_{j}}\right)^{2} .
$$

The coordinate frame $L_{j}$ is initiated together with the line and is equal to the ego vehicles coordinate frame at the moment the line is created. Unlike the ego vehicle's frame it is fixed to the world frame, i.e., $r_{O_{L_{j}} O_{W}}^{W}$ and $\psi_{L_{j} W}$ are constant and it does not follow the vehicles movement.

The state of the line $\mathcal{L}_{j}$ is

$$
\mathbf{x}_{\mathcal{L}_{j}}=\left[\begin{array}{lllll}
a_{0, j} & a_{1, j} & a_{2, j} & s_{j} & e_{j}
\end{array}\right]^{T},
$$

where $s_{j}$ and $e_{j}$ are the start and end points of the line given as scalar $x^{L_{j}}$ values.

The motion model of the stationary objects in the form

$$
\mathbf{x}_{t+1}=F \mathbf{x}_{t}+w_{t}, \quad w_{t} \sim \mathcal{N}(0, Q),
$$

is simple, since the objects are not moving. For the points, the system matrix, referred to as $F_{\mathcal{P}}$, is the identity matrix. The term $w_{t}$ in (7) represents the process noise. We include some dynamics into the motion model of the line. We assume that the lines are shrinking with a factor $\lambda<1$ according to

$$
\begin{aligned}
& s_{j, t+1}=s_{j, t}+\lambda\left(e_{j, t}-s_{j, t}\right), \\
& e_{j, t+1}^{j}=e_{j, t}-\lambda\left(e_{j, t}-s_{j, t}\right),
\end{aligned}
$$

leading to

$$
F_{\mathcal{L}}=\left[\begin{array}{ccccc}
1 & 0 & 0 & 0 & 0 \\
0 & 1 & 0 & 0 & 0 \\
0 & 0 & 1 & 0 & 0 \\
0 & 0 & 0 & 1-\lambda & \lambda \\
0 & 0 & 0 & \lambda & 1-\lambda
\end{array}\right]
$$

This shrinking behavior for the lines allows us to automatically adjust the starting and end points of the lines according to the incoming measurements. 


\section{B. Measurement Model}

The measurement equation describing the measurements relation to a point $\mathcal{P}_{i}$ is defined as

$$
\mathbf{y}_{\mathcal{P}_{i}, t}=\mathbf{x}_{\mathcal{P}_{i}, t}+v_{\mathcal{P}, t}, \quad v_{\mathcal{P}, t} \sim \mathcal{N}\left(0, R_{\mathcal{P}}\right),
$$

where the output $\mathbf{y}_{\mathcal{P}_{i}, t}=\mathcal{S}_{m}^{W}$ is the observation $m$ in the world coordinate frame associated to the $i^{\text {th }}$ point. The term $v_{\mathcal{P}, t}$ in (10) represents the measurement noise associated with the radar. The measurement equation describing the measurements relation to a line $\mathcal{L}_{j}$ is

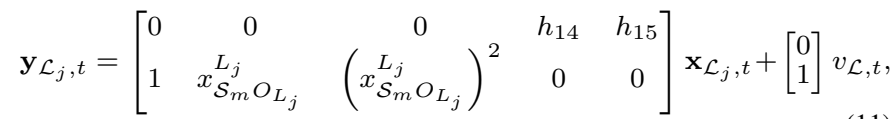

where $\mathbf{y}_{\mathcal{L}_{j}, t}=\mathcal{S}_{m}^{L_{j}}$ is the observation $m$ in the $L_{j}$ coordinate frame and associated to line $\mathcal{L}_{j}$. The term $v_{\mathcal{L}, t} \sim \mathcal{N}\left(0, R_{\mathcal{L}}\right)$ represents the measurement noise. The first row of the measurement matrix, which determines the update of the start and the end points, depends on the position of the observation in relation to the predictions of the start and the end points according to

$$
\left[\begin{array}{ll}
h_{14} & h_{15}
\end{array}\right]= \begin{cases}{\left[\begin{array}{ll}
1 & 0 \\
{[0} & 1 \\
0 & 0
\end{array}\right]} & \begin{array}{l}
\text { if } x_{\mathcal{S}_{m} O_{L_{j}}}^{L_{j}} \leq s_{j, t \mid t-1} \\
0
\end{array} x_{\mathcal{S}_{m} O_{L_{j}}}^{L_{m}} \geq e_{j, t \mid t-1} \\
\text { otherwise. }\end{cases}
$$

This type of measurement where some measured quantities $\left(x_{\mathcal{S}_{m} O_{L_{j}}}^{L_{j}}\right.$ in our case) appear as model parameters is not conventional in dynamic estimation literature and can be considered as an extension of the so-called "errors in variables" framework. In our application, this enables us to use the Kalman filter because the resulting model is linear.

\section{Data Association and Gating}

At every sampling time, the system receives a batch of $n_{\mathcal{S}}$ observations $\mathcal{S}_{m}, m=1, \ldots, n_{\mathcal{S}}$ from the radar. These new measurements can be associated to existing tracked points $\mathcal{P}_{i}$, $i=1, \ldots, n_{\mathcal{P}}$ or to tracked lines $\mathcal{L}_{j}, j=1, \ldots, n_{\mathcal{L}}$, or a new track is initiated. The number of association events (hypotheses) is extremely large. The classical technique to reduce the number of these hypotheses is called gating [21]. We apply gating and make a nearest-neighbor type data association based on likelihood ratio tests. Other more complicated data association methods like multiple hypothesis tracking [22] or joint probabilistic data association [21] can also be used in our framework. However, these are quite complicated and computationally costly approaches and the nearest neighbor type algorithm we used has been found to give sufficient performance for our case. The gating and the data association are performed according to the following calculations. The likelihood $\ell_{\mathcal{S}_{m}, \mathcal{P}_{i}}$ that the observation $\mathcal{S}_{m}$ corresponds to the $i^{\text {th }}$ point $\mathcal{P}_{i}$ is given by

$$
\ell_{\mathcal{S}_{m} \mathcal{P}_{i}}= \begin{cases}\mathcal{N}\left(\mathcal{S}_{m} ; \hat{\mathbf{y}}_{\mathcal{P}_{i}, t \mid t-1}, S_{\mathcal{P}_{i}}\right), & \text { if } \mathcal{S}_{m} \in \mathcal{G}_{\mathcal{P}_{i}} \\ 0, & \text { otherwise }\end{cases}
$$

where $\hat{\mathbf{y}}_{\mathcal{P}_{i}, t \mid t-1}$ is the predicted measurement of the point $\mathcal{P}_{i}$ according to the model (10) and $S_{\mathcal{P}_{i}, t \mid t-1}$ is its covariance (innovation covariance) in the Kalman filter. The gate $\mathcal{G}_{\mathcal{P}_{i}}$ is defined as the region

$$
\mathcal{G}_{\mathcal{P}_{i}} \triangleq\left\{\mathbf{y} \mid\left(\mathbf{y}-\hat{\mathbf{y}}_{\mathcal{P}_{i}, t \mid t-1}\right)^{T} S_{\mathcal{P}_{i}, t \mid t-1}^{-1}\left(\mathbf{y}-\hat{\mathbf{y}}_{\mathcal{P}_{i}, t \mid t-1}\right) \leq \delta_{\mathcal{P}}\right\}
$$

where $\delta_{\mathcal{P}}$ is the gating threshold.

The likelihood that the observation $m$ corresponds to the $j^{\text {th }}$ line state is derived by considering the orthogonal distance between the line and the observation. To simplify the calculations we assume that the curvature of the line is small and that the orthogonal distance can be approximated with the $y$ distance between the observation and the line expressed using the lines coordinate frame $L_{j}$, i.e.,

$$
\epsilon_{\mathcal{S}_{m} \mathcal{L}_{j}}=y_{\mathcal{S}_{m} O_{L_{j}}}^{L_{j}}-\hat{y}_{\mathcal{S}_{m} O_{L_{j}}}^{L_{j}}
$$

where

$$
\hat{y}_{\mathcal{S}_{m} O_{L_{j}}}^{L_{j}} \triangleq\left[\begin{array}{lllll}
1 & x_{\mathcal{S}_{m} O_{L_{j}}}^{L_{j}} & \left(x_{\mathcal{S}_{m} O_{L_{j}}}^{L_{j}}\right)^{2} & 0 & 0
\end{array}\right] \hat{\mathbf{x}}_{\mathcal{L}_{j}, t \mid t-1} .
$$

The likelihood $\ell_{\mathcal{S}_{m} \mathcal{L}_{j}}$ that the observation corresponds to the $j^{\text {th }}$ line is then given by

$$
\ell_{\mathcal{S}_{m} \mathcal{L}_{j}}= \begin{cases}\mathcal{N}\left(\epsilon_{\mathcal{S}_{m} \mathcal{L}_{j}} ; 0, \mathrm{E}\left(\Delta_{y_{j}}^{2}\right)\right), & \text { if }\left[\begin{array}{c}
x_{\mathcal{S}_{m} O_{L_{j}}}^{L_{j}} \\
y_{\mathcal{S}_{m} O_{L_{j}}}^{L_{j}}
\end{array}\right] \in \mathcal{G}_{\mathcal{L}_{j}} \\
0, & \text { otherwise }\end{cases}
$$

where $y_{j}=y_{\mathcal{S}_{m} O_{L_{j}}}^{L_{j}}$ and the gate $\mathcal{G}_{\mathcal{L}_{j}}$ is defined as

$$
\begin{array}{r}
\mathcal{G}_{\mathcal{L}_{j}} \triangleq\left\{\left[\begin{array}{l}
x \\
y
\end{array}\right] \mid\left(y-\hat{y}_{\mathcal{S}_{m} O_{L_{j}}}^{L_{j}}\right)^{2} \mathrm{E}\left(\Delta_{y_{j}}^{2}\right)^{-1} \leq \delta_{\mathcal{L}},\right. \\
\left.s_{j}-\delta_{s}<x<e_{j}+\delta_{e}\right\} .
\end{array}
$$

In (17) and (18), $\mathrm{E}\left(\Delta_{y_{j}}^{2}\right)$ represents the uncertainty of the line in the $y$ direction at the $x$-value $x_{\mathcal{S}_{m} O_{L_{j}}}^{L_{j}}$. This covariance has to be calculated in terms of the state estimate $\hat{\mathbf{x}}_{\mathcal{L}_{j}, t \mid t-1}$ and its covariance $P_{\mathcal{L}_{j}, t \mid t-1}$. This derivation can be made by first rewriting the line equation (6) with mean parameters and a deviation $\Delta$

$$
y+\Delta_{y}=\left(a_{0}+\Delta_{a_{0}}\right)+\left(a_{1}+\Delta_{a_{1}}\right) x+\left(a_{2}+\Delta_{a_{2}}\right) x^{2},
$$

where the superscripts and subscripts are discarded for the sake of brevity. This gives

$$
\Delta_{y}=\Delta_{a_{0}}+\Delta_{a_{1}} x+\Delta_{a_{2}} x^{2} .
$$

Considering the squared expectation of this deviation, we obtain

$$
\begin{aligned}
& \mathrm{E}\left(\Delta_{y}^{2}\right)=\mathrm{E}\left(\Delta_{a_{0}}+\Delta_{a_{1}} x+\Delta_{a_{2}} x^{2}\right)^{2} \\
& =\mathrm{E}\left(\begin{array}{lll}
\left(\begin{array}{ccc}
1 & x & x^{2}
\end{array}\right)\left(\begin{array}{lll}
\Delta_{a_{0}} & \Delta_{a_{1}} & \Delta_{a_{2}}
\end{array}\right)^{T} \\
\quad \times\left(\begin{array}{lll}
\Delta_{a_{0}} & \Delta_{a_{1}} & \Delta_{a_{2}}
\end{array}\right)\left(\begin{array}{ccc}
1 & x & x^{2}
\end{array}\right)^{T}
\end{array}\right) \\
& =\left(\begin{array}{lll}
1 & x & x^{2}
\end{array}\right) \mathrm{E}\left(\left(\begin{array}{ccc}
\Delta_{a_{0}} & \Delta_{a_{1}} & \Delta_{a_{2}}
\end{array}\right)^{T}\right. \\
& \quad \times\left(\begin{array}{llll}
\Delta_{a_{0}} & \Delta_{a_{1}} & \Delta_{a_{2}}
\end{array}\right)\left(\begin{array}{ccc}
1 & x & x^{2}
\end{array}\right)^{T} .
\end{aligned}
$$


Now, the expectation above is given by the upper-left $3 \times 3$ partition of the covariance matrix $P_{\mathcal{L}_{j}, t \mid t-1}$ which we denote by $P_{\mathcal{L}_{j}, t \mid t-1}^{3 \times 3}$. Hence,

$$
\begin{aligned}
\mathrm{E}\left(\Delta_{y_{j}}^{2}\right)= & \left(\begin{array}{lll}
1 & x_{\mathcal{S}_{m} O_{L_{j}}}^{L_{j}} & \left(x_{\mathcal{S}_{m} O_{L_{j}}}^{L_{j}}\right)^{2}
\end{array}\right) P_{\mathcal{L}_{j}, t \mid t-1}^{3 \times 3} \\
& \times\left(\begin{array}{lll}
1 & x_{\mathcal{S}_{m} O_{L_{j}}}^{L_{j}} & \left(x_{\mathcal{S}_{m} O_{L_{j}}}^{L_{j}}\right)^{2}
\end{array}\right)^{T} .
\end{aligned}
$$

Having calculated the likelihood values, we form two matrices of likelihood values, one matrix $\Gamma_{\mathcal{P}} \in \mathbb{R}^{n_{\mathcal{S}} \times n_{\mathcal{P}}}$ with the combinations of observations and points, according to (13), and one matrix $\Gamma_{\mathcal{L}} \in \mathbb{R}^{n_{\mathcal{S}} \times n_{\mathcal{L}}}$ with the combinations of observations and lines, according to (17).

First we find the the maximum value of $\Gamma_{\mathcal{P}}$, and call the corresponding point state $i_{m}$ and measurement $m_{m}$. Thereafter we find the maximum value of the $m^{\text {th }}$ row, corresponding to measurement $m_{m}$ of matrix $\Gamma_{\mathcal{L}}$ and call the corresponding line state $j_{m}$. The likelihood ratio denoted by $\Lambda\left(\mathcal{S}_{m}\right)$ is now given by

$$
\Lambda\left(\mathcal{S}_{m}\right) \triangleq \frac{\ell_{S_{m} \mathcal{P}_{i_{m}}}}{\ell_{S_{m} \mathcal{L}_{j_{m}}}}
$$

The corresponding likelihood ratio test is

$$
\Lambda\left(\mathcal{S}_{m}\right) \underset{H_{1}}{\stackrel{H_{0}}{\gtrless}} \eta
$$

where $H_{0}$ and $H_{1}$ corresponds to hypotheses that the measurement $\mathcal{S}_{m}$ is associated to the point $\mathcal{P}_{i_{m}}$ and to the line $\mathcal{L}_{j_{m}}$, respectively. The threshold is selected as $\eta<1$, since (13) is two dimensional and (17) is one dimensional. More theory about likelihood test is given in e.g. [23].

No two measurements may originate from the same point source and no two sources may give rise to the same measurements. However, one line source may give rise to multiple measurements. This means that if measurement $\mathcal{S}_{m}$ is associated to point $\mathcal{P}_{i}$, then the values in the $m^{\text {th }}$ row of the two matrices as well as the $i^{\text {th }}$ column of the point likelihood matrix must be set to zero to exclude the measurement and the point from further association. However, if $\mathcal{S}_{m}$ is associated to line $\mathcal{L}_{j}$, then only the values in the $m^{\text {th }}$ rows of the two matrices are set to zero because the line $\mathcal{L}_{j}$ can still be associated to other measurements. The procedure is repeated until all measurements with non-zero likelihood have been associated to either a point or a line. A new point is initiated if the observations could not be associated to an existing state. This is true when a measurement is not in the gate of a nonassociated point or a line.

\section{HANDLING TRACKS}

A line is initiated from tracked points under the assumption that a number of points form a line parallel to the road. In this section we will discuss track handling matters such as initiating and removing tracks.

\section{A. Initiating Lines}

All points $\mathcal{P}_{i}$ are transformed into the ego vehicles coordinate frame since the road's geometry is given in this frame. The road geometry is described by the polynomial given in (1). We consider hypothetical lines passing through each point $\mathcal{P}_{k}$ parallel to the road. For each such line, the corresponding lateral distance $l_{\mathcal{P}_{k}}$ is given by

$$
l_{\mathcal{P}_{k}}=\hat{y}_{\mathcal{P}_{k} O_{E}}^{E}-\delta_{r} \hat{x}_{\mathcal{P}_{k} O_{E}}^{E}-\frac{c_{0}}{2}\left(\hat{x}_{\mathcal{P}_{k} O_{E}}^{E}\right)^{2} .
$$

The likelihood $\ell_{\mathcal{P}_{i} \mathcal{P}_{k}}$ that a point $\mathcal{P}_{i}$ is on the line of point $\mathcal{P}_{k}$ is then given by

$$
\ell_{\mathcal{P}_{i} \mathcal{P}_{k}}= \begin{cases}\mathcal{N}\left(\epsilon_{\mathcal{P}_{i} \mathcal{P}_{k}} ; 0, P_{\mathcal{P}_{k},(2,2)}^{E}\right), & \text { if }\left[\begin{array}{c}
\hat{x}_{\mathcal{P}_{i}}^{L_{E} O_{E}} \\
\hat{y}_{\mathcal{P}_{i} O_{E}}^{L}
\end{array}\right] \in \mathcal{G}_{\mathcal{P}_{k}} \\
0, & \text { otherwise }\end{cases}
$$

where the lateral distance between the point $\mathcal{P}_{i}$ and the proposed new line of point $\mathcal{P}_{k}$ is given by

$$
\epsilon_{i k}=\hat{y}_{\mathcal{P}_{i} O_{E}}^{E}-\hat{y}_{\mathcal{P}_{k} O_{E}}^{E},
$$

where

$$
\hat{y}_{\mathcal{P}_{k} O_{E}}^{E}=l_{\mathcal{P}_{k}}+\delta_{r} \hat{x}_{\mathcal{P}_{i} O_{E}}^{E}+\frac{c_{0}}{2}\left(\hat{x}_{\mathcal{P}_{i} O_{E}}^{E}\right)^{2},
$$

and the state covariance in the ego vehicles coordinate frame is given by

$$
P_{\mathcal{P}_{k}}^{E}=\left(R^{E W}\right)^{T} P_{\mathcal{P}_{k}} R^{E W} .
$$

The notation $P_{\mathcal{P}_{k},(2,2)}^{E}$ refers to the lower-right element, i.e., the variance in the diagonal corresponding to $y^{E}$. The gate $\mathcal{G}_{\mathcal{P}_{k}}$ is defined as

$$
\begin{aligned}
\mathcal{G}_{\mathcal{P}_{k}} \triangleq\left\{\left[\begin{array}{l}
x \\
y
\end{array}\right] \mid \frac{\left(y-\hat{y}_{\mathcal{P}_{k} O_{E}}^{E}\right)^{2}}{P_{\mathcal{P}_{k},(2,2)}^{E}}\right. & \leq \delta_{\mathcal{L}}, \\
-\delta_{s} & \left.<x-\hat{x}_{\mathcal{P}_{k} O_{E}}^{E}<\delta_{e}\right\} .
\end{aligned}
$$

From all combinations of likelihoods we form a symmetric matrix $\Gamma_{\mathcal{I}}$. The columns of $\Gamma_{\mathcal{I}}$ are summed and the maximum value corresponding to column $k_{m}$ is chosen. If this column contains more than a certain number $\kappa$ of non-zero rows, corresponding to points

$$
\mathcal{P}_{l}=\left\{\mathcal{P} \mid \Gamma_{\mathcal{I}}\left(:, k_{m}\right) \neq 0\right\}
$$

within the gate of $\mathcal{P}_{k_{m}}$, a line is formed from the points $\mathcal{P}_{l}$. The new line's states $a_{0}, a_{1}$ and $a_{2}$ are estimated by solving a least square problem using the points $\mathcal{P}_{l}$. The states $s$ and $e$ are the minimum and maximum $\mathrm{x}$-coordinate value of the points, respectively. All elements in column $k_{m}$ and rows $i_{m}$ are set to zero and the procedure is repeated until no column contains more than $\kappa$ non-zero elements.

\section{B. Remove Lines or Points}

For each state we introduce a counter. The counter is increased if the state is updated with new measurements and decreased if it was not updated during one iteration. A state is removed if the counter is zero. 


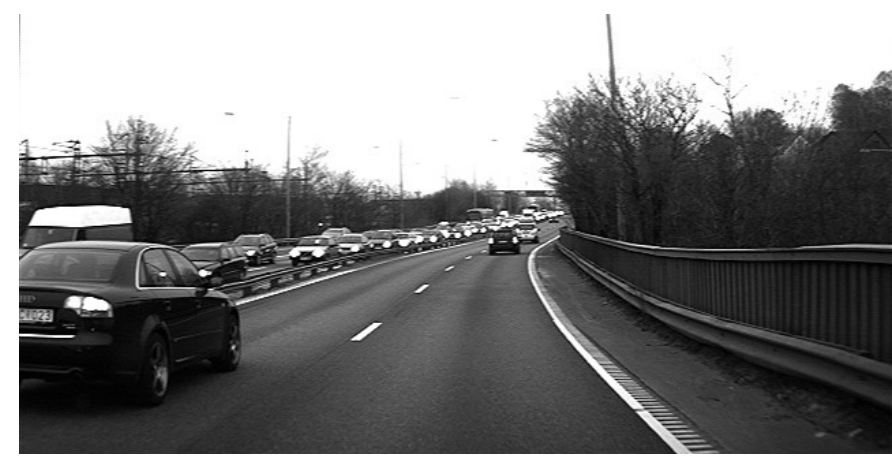

(a)

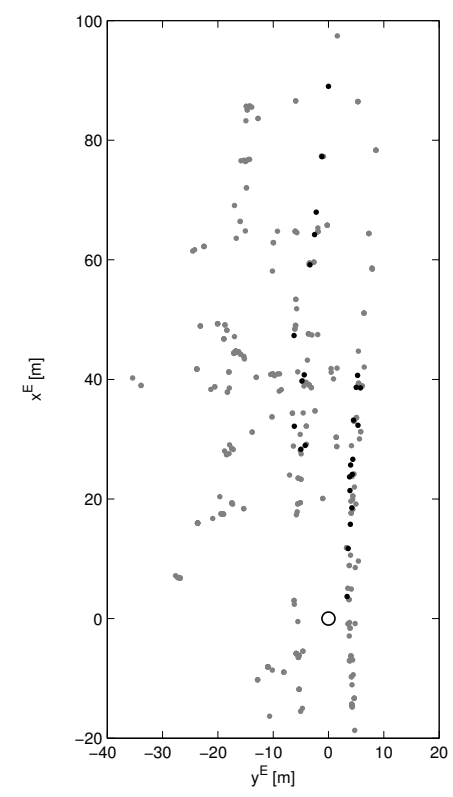

(b)

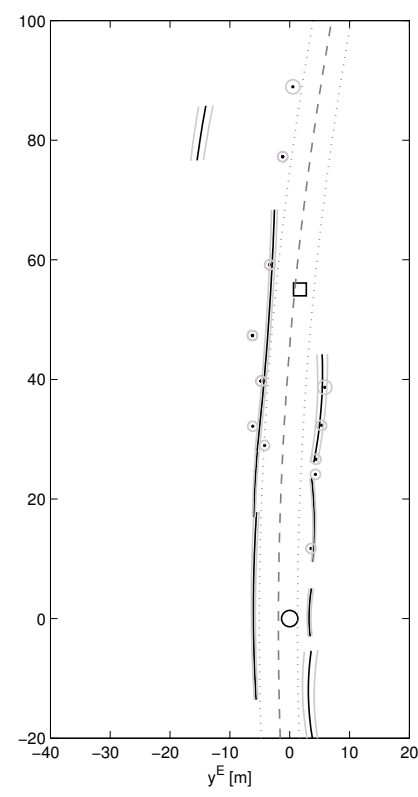

(c)
Figure 2. A traffic situation is shown in Figure (a). Figure (b) shows the radar reflections, and Figure (c) the resulting tracked points and lines. The circle is the ego vehicle, the square is the tracked vehicle in front and the dashed gray lines illustrates the tracked road curvature.

\section{EXPERIMENTS AND RESULTS}

Let us start by showing the information given by an ordinary automotive ACC radar, for the traffic situation shown in Figure 2a. The ego vehicle, indicated by a circle, is situated at the $(0,0)$-position in Figure $2 \mathrm{~b}$, and the black dots are the radar reflections, or stationary observations, at one time sample. The gray dots are former radar reflections, obtained at earlier time samples. Figure 2c shows the estimated points and lines for the same scenario. The mean values of the states are indicated by solid black lines or points. Furthermore, the state variance, by means of the $1 \sigma$ confidence interval, is illustrated by gray lines or ellipses, respectively. In [16] the authors presented a new approach to estimate the road curvature (1), which we show here as gray dashed lines. We also show the tracked vehicle in front of the ego vehicle illustrated by a square.

In Figure 3a we see a traffic scenario with a highway exit. The corresponding bird's eye view is shown in Figure $3 b$. The origin of the line's coordinate systems are illustrated with dots and a number which is repeated at each line. Line 1 indicates the guardrail to the right of the exit, line 2 is the

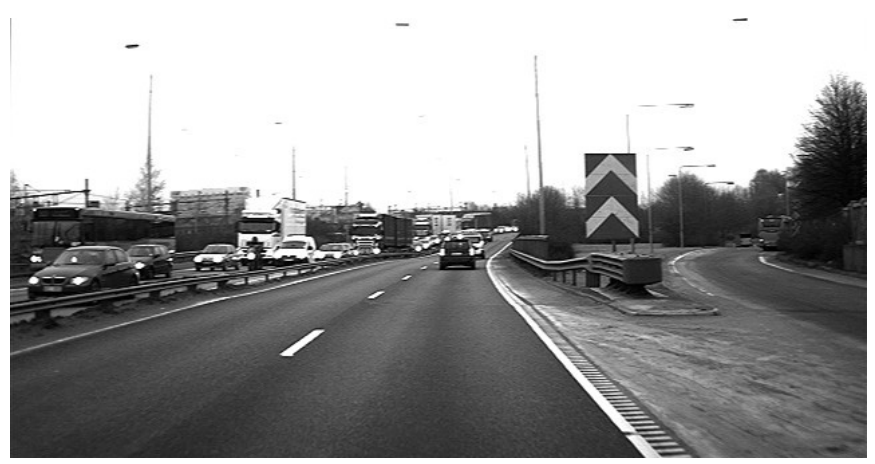

(a)

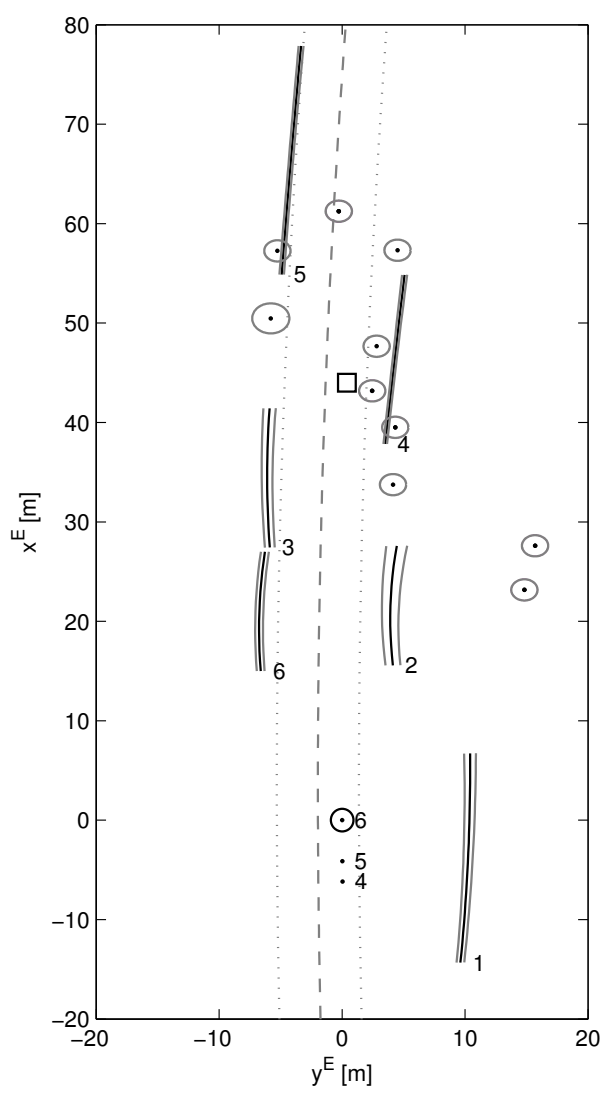

(b)

Figure 3. Highway exit with guardrails, the camera view is shown in Figure (a) and the bird's eye view with the estimated states in Figure (b).

guardrail starting at the exit sign. The gap between line 3 and line 5 is probably due to the dimple, where the radar signals are transmitted above the guard rail, hence not giving us any stationary observations in the desired region.

Our last example shows a situation from a rural road, see Figure 4a. The lines 5 and 6 are the guardrails of a bridge. Line 4 depicts a fence behind the bridge. From the camera view it is hard to recognize and also the radar has problems to track it, indeed the gray lines indicates a large uncertainty for this case.

\section{CONCLUSION}

In this contribution we have derived a method for tracking stationary objects as extended objects using radar measurements. Typically radar echoes stem from delineators or 


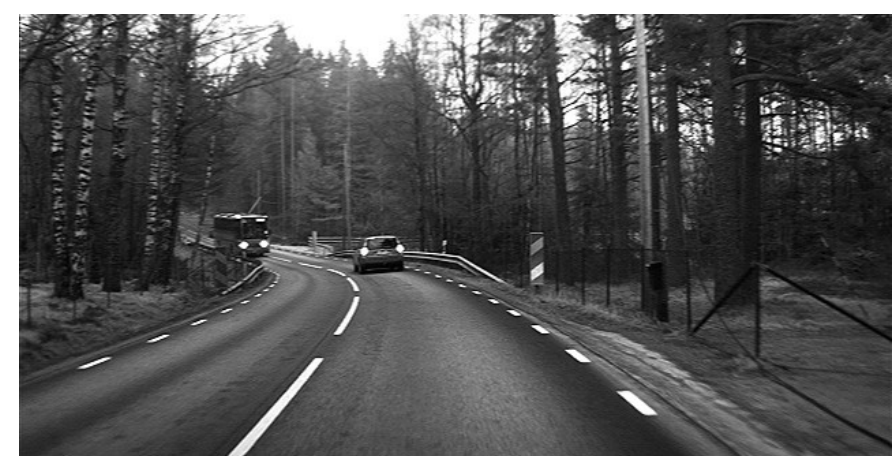

(a)

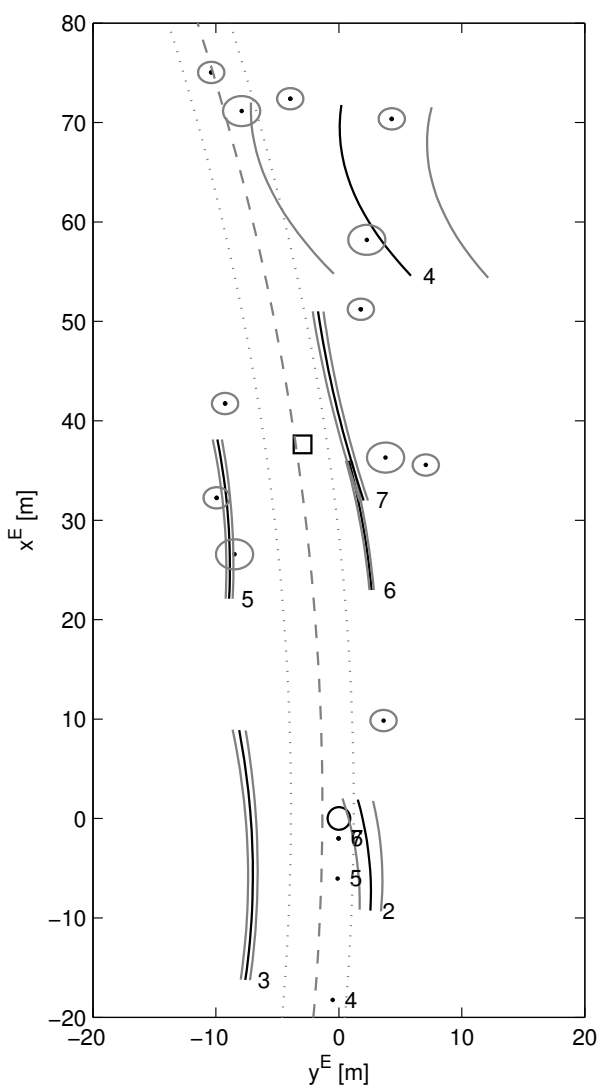

(b)

Figure 4. A traffic scenario from a rural road, with guardrails on both sides of a bridge is shown. Note that the fence behind the bridge in Figure (a) is illustrated by line 4 in Figure (b), observe the large uncertainty.

guardrails, which are tracked as points or lines, respectively, in a standard Kalman filter framework. A major part of the present approach is the data association and gating problem. The approach has been evaluated on real and relevant data from both highways and rural roads in Sweden. The results are not perfect, but surprisingly good at times, and of course much more informative than just using raw measurements. Furthermore, the standard state representation of the objects should not be underestimated since it is compact and easy to send on a vehicle CAN-bus.

\section{ACKNOWLEDGMENT}

The authors would like to thank the SEnsor Fusion for Safety (SEFS) project within the Intelligent Vehicle Safety
Systems (IVSS) program and the strategic research center MOVIII, funded by the Swedish Foundation for Strategic Research (SSF) for financial support.

\section{REFERENCES}

[1] M. Buehler, K. Iagnemma, and S. Singh, Eds., Special Issue on the 2007 DARPA Urban Challenge, Part I. Journal of Field Robotics, 2008, vol. $25(8)$.

[2] - Special Issue on the 2007 DARPA Urban Challenge, Part II Journal of Field Robotics, 2008, vol. 25 (9)

[3] — Special Issue on the 2007 DARPA Urban Challenge, Part III Journal of Field Robotics, 2008, vol. 25 (10).

[4] B. Ristic, S. Arulampalam, and N. Gordon, Beyond the Kalman Filter: Particle filters for tracking applications. London, UK: Artech House, 2004.

[5] K. Gilholm and D. Salmond, "Spatial distribution model for tracking extended objects," in IEE Proceedings of Radar, Sonar and Navigation, vol. 152, no. 5, October 2005, pp. 364-371.

[6] J. Vermaak, N. Ikoma, and S. J. Godsill, "Sequential Monte Carlo framework for extended object tracking," IEEE Proceedings of Radar Sonar and Navigation, vol. 152, no. 5, pp. 353-363, Oct. 2005.

[7] D. Angelova and L. Mihaylova, "Extended object tracking using Monte Carlo methods," IEEE Transactions on Signal Processing, vol. 56, no. 2 , pp. 825-832, Feb. 2008.

[8] J. W. Koch, "Bayesian approach to extended object and cluster tracking using random matrices," IEEE Transactions on Aerospace and Electronic Systems, vol. 44, no. 3, pp. 1042-1059, Jul. 2008.

[9] A. Kirchner and T. Heinrich, "Model based detection of road boundaries with a laser scanner," in Proceedings of the IEEE International Conference on Intelligent Vehicles, Stuttgart, Germany, 1998, pp. 93-98.

[10] A. Polychronopoulos, A. Amditis, N. Floudas, and H. Lind, "Integrated object and road border tracking using $77 \mathrm{GHz}$ automotive radars," in IEE Proceedings of Radar, Sonar and Navigation, vol. 151, no. 6, Dec. 2004, pp. 375-381.

[11] C. Lundquist and T. B. Schön, "Estimation of the free space in front of a moving vehicle," in Proceedings of the SAE World Congress, Detroit, MI, USA, Apr. 2009, accepted for publication.

[12] W. S. Wijesoma, K. R. S. Kodagoda, and A. P. Balasuriya, "Roadboundary detection and tracking using ladar sensing," IEEE Transactions on Robotics and Automation, vol. 20, no. 3, pp. 456-464, June 2004.

[13] K. R. S. Kodagoda, W. S. Wijesoma, and A. P. Balasuriya, "CuTE: Curb Tracking and Estimation," IEEE Transactions on Control Systems Technology, vol. 14, no. 5, pp. 951-957, Sept. 2006.

[14] B. Fardi, U. Scheunert, H. Cramer, and G. Wanielik, "Multi-modal detection and parameter-based tracking of road borders with a laser scanner," in Proceedings of the IEEE Intelligent Vehicles Symposium, June 2003, pp. 95-99.

[15] Y. Wang, L. Bai, and M. Fairhurst, "Robust road modeling and tracking using condensation," IEEE Transactions on Intelligent Transportation Systems, vol. 9, no. 4, pp. 570-579, Dec. 2008.

[16] C. Lundquist and T. B. Schön, "Road geometry estimation and vehicle tracking using a single track model," in Proceedings of the IEEE Intelligent Vehicle Symposium, Eindhoven, The Netherlands, Jun. 2008, pp. 144-149.

[17] Z. Zomotor and U. Franke, "Sensor fusion for improved vision based lane recognition and object tracking with range-finders," in Proceedings of IEEE Conference on Intelligent Transportation System, Boston, MA, USA, Nov. 1997, pp. 595-600.

[18] A. Gern, U. Franke, and P. Levi, "Advanced lane recognition - fusing vision and radar," in Proceedings of the IEEE Intelligent Vehicles Symposimum, Dearborn, MI, USA, Oct. 2000, pp. 45-51.

[19] - "Robust vehicle tracking fusing radar and vision," in Proceedings of the international conference of multisensor fusion and integration for intelligent systems, Baden-Baden, Germany, Aug. 2001, pp. 323-328.

[20] R. E. Kalman, "A new approach to linear filtering and prediction problems," Transactions of the ASME, Journal of Basic Engineering, vol. 82, pp. 35-45, 1960.

[21] Y. Bar-Shalom and T. E. Fortmann, Tracking and Data Association, ser. Mathematics in science and engineering. Orlando, FL, USA: Academic Press, 1988 .

[22] D. B. Reid, "An algorithm for tracking multiple targets," IEEE Transactions on Automatic Control, vol. 24, no. 6, pp. 84-90, 1979.

[23] H. L. van Trees, Detection, Estimation, and Modulation Theory. New York, USA: John Wiley \& Sons, 1968. 